\title{
Nesprin-1 plays an important role in the proliferation and apoptosis of mesenchymal stem cells
}

\author{
WENGANG YANG, HUI ZHENG, YONGYI WANG, FENG LIAN, ZHENGLEI HU and SONG XUE \\ Department of Cardiovascular Surgery, Renji Hospital, Shanghai Jiaotong University \\ School of Medicine, Shanghai 200127, P.R. China
}

Received April 10, 2013; Accepted June 27, 2013

DOI: $10.3892 / \mathrm{ijmm} .2013 .1445$

\begin{abstract}
The aim of this study was to investigate the expression of nesprin-1 protein and its effects on rat bone marrow mesenchymal stem cells (MSCs). MSCs were cultured in DMEM and surface-associated antigens of MSCs were detected by flow cytometry. The protein expression of nesprin-1 was detected by immunofluorescence and western blot analysis. A lentiviral vector expressing small interfering RNA (siRNA) targeting nesprin-1 was constructed (LV-siNesprin-1) and the MSCs were subsequently transfected with this vector. Another group of MSCs was transfected with the LV-GFP vector and another group of untransfected cells was used as the controls (normal group). The protein expression level of nesprin-1 in the 3 groups of MSCs (LV-siNesprin-1, LV-GFP and normal group) was measured by western blot analysis. Cell proliferation was assessed by MTT assay, and the cell cycle and apoptosis were detected by flow cytometry. DAPI was used to stain the nucleus of the MSCs. The MSCs appeared spindle-shaped with irregular processes and were positive for CD90, CD29 and negative for CD45. Nesprin-1 protein was found in the nuclear membrane. The protein expression of nesprin-1 in the LV-siNesprin-1 group was lower than that in the LV-GFP $(\mathrm{P}=0.03)$ and normal group $(\mathrm{P}=0.028)$; this difference was significant $(\mathrm{P}<0.05)$. The cell proliferation of the MSCs transfected with LV-siNesprin-1 was reduced; the apoptotic rate was higher in the LV-siNesprin-1 group compared with the other 2 groups (LV-GFP and normal group) $(\mathrm{P}=0.032, \mathrm{P}=0.025$, respectively; $\mathrm{P}<0.05)$. The changes in the morphology of the nucleus in the LV-siNesprin-1 group included fusion and fragmentation. In conclusion, the data presented in this study indicate that nesprin-1 regulates the proliferation and apoptosis of MSCs; our results are consistent
\end{abstract}

Correspondence to: Professor Song Xue, Department of Cardiovascular Surgery, Renji Hospital, Shanghai Jiaotong University School of Medicine, 1630 Dongfang Road, Shanghai 200127, P.R. China

E-mail: xuesong64@163.com

Key words: mesenchymal stem cells, nesprin-1, proliferation, apoptosis with those from previous studies. Thus, nesprin-1 protein plays an important role in the proliferation and apoptosis of MSCs.

\section{Introduction}

Nesprin-1 is a protein isoform of the nesprin protein family that contains spectrin repeats similar to those in mAKAP, which forms homodimers and specifically targets the nuclear envelope through a KASH domain (1-5). Nesprin-1 $\alpha$ is also a candidate for a mAKAP nuclear envelope receptor. It has been reported that mutations in the nesprin-1 gene may be responsible for adult cerebella ataxia and mutations of nesprin-1 which interact with lamin A/C may lead to at least 2 distinct human disease phenotypes, myopathic or neurologic, a feature similar to that found in laminopathies. Puckelwartz et al (6) reported $\Delta / \Delta \mathrm{KASH}$ mice expresses nesprin-1 without its carboxyl-terminal KASH domain; these $\Delta / \Delta \mathrm{KASH}$ mice have a normally assembled but dysfunctioning nuclear membrane complex and provide a model for nesprin-1 mutations and developing cardiomyopathy with associated cardiac conduction system disease.

Mesenchymal stem cells (MSCs) are bone marrow-derived cells that retain the capability to differentiate into various types of tissue cells and contribute to the regeneration of a variety of tissues, including bone, cartilage, muscle and adipose tissue (7-9). MSCs, after being transplantated into the ischemic myocardial tissue, secrete a variety of factors including vascular endothelial growth factor (VEGF). The cardioprotective effects of MSCs are known to be mediated not only by their differentiation into cardiomyocyte-like cells, but also by their ability to supply large amounts of angiogenic, antiapoptotic and mitogenic factors (10-12). These findings suggest the therapeutic potential of MSCs for heart failure.

From the literature, we know that nesprin proteins exist only in multiple tissues (skeletal, cardiac and vascular smooth muscle) and not in stem cells (2). The study of nesprin-1 protein, which is speculated to be localized to the nuclear membrane, may aid in the understanding of the process through which MSCs differentiate into cardiomyocyte-like cells.

\section{Materials and methods}

Animals. Clean Sprague-Dawley (SD) rats, weighing 250-300 g, were obtained from the Experimental Animal Center of Shanghai Jiaotong University Medical School, Shanghai, 
China (production license: scxk (hu)2004-0001; use license no. syxk (hu)2003-2009). The present study was reviewed and approved by the University Institutional Animal Care and Use Committee.

Reagents. Except where otherwise specified, all reagents were obtained from Sigma-Aldrich Chemical Co. (St. Louis, MO, USA) and Gibco (Grand Island, NY,USA), including cell culture medium [low-glucose Dulbecco's modified Eagle's medium (DMEM), fetal bovine serum (FBS)]. The cardiac-specific antibodies (TNI, $\alpha$-sarcomeric actin, desmin), FITC-conjugated goat anti-rat antibodies (CD45), PE-conjugated rabbit anti-rat antibodies (CD90), allophycocyanin (APC)-conjugated rabbit anti-rat antibodies (CD29) and FITC-conjugated rabbit anti-rat nesprin- 1 antibodies, were purchased from Abcam (Cambridge, UK). The BLOCK-iT ${ }^{\text {TM }}$ POLIImiR RNAi Expression Vector kit with EmGFP, pcDNA ${ }^{\mathrm{TM}}$ 6.2-GW/EmGFPmiR, Escherichia coli (E. coli) DH5, Lipofectamine ${ }^{\circledR} 2000$, Opti-MEM, TRIzol reagent and pLenti6.3/V5-DEST were purchased from Invitrogen (Carlsbad, CA, USA). Restriction endonuclease, DNA ligase and the Large Plasmid DNA Extraction kit were purchased from Qiagen (Dusseldorf, Germany). The reagents and instruments for immunohistochemistry, immunofluorescence and western blot analysis were purchased from Gibco, Sigma-Aldrich Chemical Co. and Invitrogen, respectively.

Cell culture. Eight-week-old SD rats (250-300 g) were prepared as donors. The procedures were performed in accordance with the guidelines for animal experimentation of Shanghai Jiaotong University and approved by the institutional ethics committee. Under general anesthesia with ether (approximately $100 \mu \mathrm{l}$ ) bone marrow was aspirated from both the tibia and femur with a 20 -gauge needle attached to a $10-\mathrm{ml}$ syringe containing $0.5 \mathrm{ml}$ DMEM with $40 \mathrm{U} / \mathrm{ml}$ heparin.

The concentration of the cells in suspension was adjusted to $5 \times 10^{5}$ mononuclear cells $/ \mathrm{ml}$ culture medium at $37^{\circ} \mathrm{C}$ in a humidified atmosphere with $5 \% \mathrm{CO}_{2}$; the cells were then seeded on culture plates, without removal of the red blood cells. Since bone marrow-derived MSCs (BMSCs) grow initially in colonies and do not reach confluence over the entire culture dish, the cells were passaged 7 days after seeding, when half the colonies reached $70-80 \%$ confluence; the cells were then passaged weekly when the cells reached confluence. For subcultures, adherent BMSCs wer harvested using 0.125\% trypsin and plated at a ratio of 1:3.

For flow cytometry, the cells were detached using accutase instead of trypsin, in order to achieve a better preservation of the cell surface molecules. 293T cells were maintained in MEM supplemented with $5 \% \mathrm{FBS}$ and $50 \mathrm{mg} / \mathrm{ml}$ gentamycin. The cells were trypsinized by a $0.05 \%$ trypsin- $0.5 \mathrm{mM}$ EDTA solution.

Flow cytometry. Flow cytometry was performed using a FACSAria flow cytometer/cell sorter (BD Biosciences, San Jose, CA, USA). Following accutase treatment, the cells were resuspended at a density of $1 \times 10^{5}$ cells $/ 200 \mu 1$ phosphate-buffered saline (PBS) and incubated with 2\% FCS (PBS-FCS) on ice. The cells were stained with antibody, and incubated with FITCconjugated CD45 monoclonal antibody, PE-conjugated CD90 monoclonal antibody and APC-conjugated CD29 monoclonal antibody (at concentrations indicated by the manufacturer) for $30 \mathrm{~min}$ at $4^{\circ} \mathrm{C}$ in the dark, and then washed in PBS-FCS. After washing, the cells were analyzed in the cytometer. At least 5,000 events were analyzed for each sample. Negative controls, used to detect the unspecific bindings, included an irrelevant antibody or PBS-FCS alone. The acquired data were analyzed using Summit software (Cytomation, Inc., Fort Collins, CO, USA).

Immunofluorescence microscopy. BMSCs grown on glass coverslips were fixed by a 20 -min incubation in $4 \%$ formaldehyde (freshly prepared from paraformaldehyde), rinsed in PBS, and stored in $70 \%$ ethanol at $-20^{\circ} \mathrm{C}$. The fixed cells were blocked for $30 \mathrm{~min}$ in blocking solution (PBS supplemented with $2 \%$ goat serum, $1 \%$ BSA, $0.1 \%$ gelatin, $0.1 \%$ Triton X-100 and $0.05 \%$ Tween-10), and incubated overnight with the primary antibody (at the dilution indicated by the manufacturer) at $4^{\circ} \mathrm{C}$. After washing, the cells were incubated with the secondary antibody (FITC-conjugated anti-rat IgG for nesprin-1) for $30 \mathrm{~min}$. Finally, the coverslips were washed, mounted in glycerol and examined under an epifluorescence microscope (Olympus, Tokyo, Japan).

Western blot analysis. After washing with PBS, the BMSCs were scraped off the culture dish and transferred to centrifuge tubes. Following centrifugation at $700 \mathrm{x} g$ for $10 \mathrm{~min}$ at $4^{\circ} \mathrm{C}$, the pellets were lysed in hot Laemmli loading buffer ( $62.5 \mathrm{mmol} / \mathrm{l}$ Tris- $\mathrm{HCl}, \mathrm{pH} 6.8,2 \%$ SDS, $10 \%$ glycerol, $0.05 \%$ $\beta$-mercaptoethanol, $0.05 \%$ bromophenol blue). Equal amounts of protein extracts $(20 \mathrm{mg} / \mathrm{lane})$ were subjected to SDS-PAGE on a 5\% stacking gel and a $10 \%$ separating gel, followed by transfer of the proteins onto nitrocellulose membranes (20 min at $10 \mathrm{~V})$. After blocking in PBS containing $0.05 \%$ Triton X-100 (TBS) and 5\% FCS for $1 \mathrm{~h}$, the blots were incubated overnight with primary antibodies (rabbit anti-rat nesprin-1) at $4^{\circ} \mathrm{C}$. After washing, the membranes were incubated with the secondary antibody (HRP-conjugated goat anti rabbit $\mathrm{IgG}$ ) for $1 \mathrm{~h}$; the bound antibody was detected by ECL. $\beta$-actin was used as an internal control.

Design and cloning of small interfering RNA (siRNA) cassettes. The nesprin-1 DNA and protein sequence was according to the GenBank accession no. NM_001029909.1. The nesprin-1 gene siRNA sequence and the corresponding miRNA oligonucleotide sequence were then designed and synthesized using Ambion design software and the sequences were verified using BLAST software: (5'-CGGGAGTTGTTG ACTATGAAA-3'); the corresponding miRNA oligonucleotide sequence was as follows: nesprin-1 forward, TGCTGTTT CATAGTCAACAACTCCCGGTTTTGGCCACTGACTGA CCGGGAGTTTGACTATGAAA; and reverse, CCTGTTT CATAGTCAAACTCCCGGTCAGTCAGTGGCCAAAACC GGGAGTTGTTGACTATGAAAC. The Vector Cloning kit was then used for restructuring; the double-stranded miRNA oligonucleotide was inserted into the miRNA expression vector (Invitrogen), and the cells were transfected with miRNA plasmids infected with $E$. coli DH5. The oligonucleotides were annealed and cloned into the BglII-HindIII site. The pDONR221 vector was processed with BP recombination reaction, in order to obtain the entry vector containing the siRNA. The sequence 
of the entry vector with siRNA and the lentiviral expression vector pLenti6/V5-DEST were processed with LR recombination reaction in order to obtain the lentiviral expression vector expressing siRNA targeting nesprin-1 (LV-siNesprin-1).

Transfection. The 293 T cells were co-transfected with a plasmid expressing GFP together with a plasmid expressing siRNA specific for GFP (siGFP) at a ratio of 1:5 using FuGENE ${ }^{\circledR}$ transfection reagent (Roche, Indianapolis, IN, USA).

Lentiviral vector production. Recombinant lentiviruses were produced by the transient transfection of $293 \mathrm{~T}$ cells using the calcium-phosphate method as previously described (13-15). Infectious lentiviruses were harvested at 48 and $72 \mathrm{~h}$ posttransfection and filtered through $0.22-\mu \mathrm{m}$-pore cellulose acetate filters as previously described (13-15). Recombinant lentiviruses were concentrated by ultracentrifugation $(2 \mathrm{~h}$ at $50,000 \mathrm{x} \mathrm{g}$ ) and subsequently purified on a sucrose $20 \%$ gradient ( $2 \mathrm{~h}$ at 46,000 $\mathrm{x} \mathrm{g}$ ) as previously described (16). Vector concentrations were analyzed using an immunocapture p24-gag ELISA (Alliance; DuPont/NEN, Boston, MA, USA) as previously described (16); the concentrated suspension of the activity of the viral titer was measured $\left(1 \times 10^{6} \mathrm{TU} / \mathrm{ml}\right)$.

Protein levels were analyzed by western blot analysis (concrete steps as the former) and immunoblotting was carried out according to standard methods with rabbit moclonal antibody against GFP (Abcam) or $\beta$-actin (Sigma-Aldrich Chemical Co.). Fluorescence-activated cell-sorter analysis was carried out as previously described (17-19).

MTT cell proliferation assay. The cells were plated on 24-well plates $\left(2 \times 10^{4}\right.$ cells/well $)$ in the growth medium for the assays. The protocols for MTT assays were as previously described (20). In brief, growth medium containing $0.25 \mathrm{mg} / \mathrm{ml}$ MTT was added to each well and the cells were further incubated at $37^{\circ} \mathrm{C}$ for $20 \mathrm{~min}$, following which the medium was replaced by $0.2 \mathrm{ml} \mathrm{DMSO} /$ well. MTT dye conversion was determined by measuring the $\mathrm{OD}_{540 \mathrm{~nm}}$ of the DMSO extracts using DMSO as the blank control.

Detection of cell cycle of MSCs by low cytometry. The cells $\left(5 \times 10^{5}\right)$ were centrifugated for $5 \mathrm{~min}$ at $800 \mathrm{rpm}$, the supernatant was collected, and they were then washed with cold PBS twice and fixed with $700 \mathrm{ml} / 1$ cold ethanol at $4^{\circ} \mathrm{C}$ overnight. The ethanol was removed by centrifugation prior to detection. The cells were washed with PBS twice, then stained with $1 \mathrm{ml}$ PI (bromide tablets) at $4^{\circ} \mathrm{C}$ for $30 \mathrm{~min}$ in the dark.

Detection of MSC apoptosis by flow cytometry. The cells $\left(5 \times 10^{6}\right)$ were centrifugated for $5 \mathrm{~min}$ at $1,000 \mathrm{rpm}$, then the culture medium was discarded. They were then washed once with PBS, centrifuged and the supernatant was removed; they were then fixed with $70 \%$ cold ethanol at $4^{\circ} \mathrm{C}$ for $1-2 \mathrm{~h}$. The ethanol was then removed by centrifugation. The cells were resuspended with $3 \mathrm{ml}$ PBS for $5 \mathrm{~min}$, filtered once and centrifuged at 1,500 rpm for $5 \mathrm{~min}$. The PBS was discarded prior to detection. The cells were stained with $1 \mathrm{ml}$ PI and FITC-Annexin $\mathrm{V}$ in $4^{\circ} \mathrm{C}$ for $30 \mathrm{~min}$ in the dark.

Morphology of the nucleus following staining of MSCs with 4,6-diamidino-2-phenylindole (DAPI) for $72 h$ and transfection with LV-siNesprin-1. The cells were passaged when they became nearly confluent. Sterile DAPI solution was added to the culture medium. The MSCs were rinsed 6 times in PBS solution to remove all excess unbound DAPI. The MSCs were cultured in culture medium [DMEM, supplemented with $20 \%$ FBS and penicillin $(100 \mathrm{U} / \mathrm{ml}) /$ streptomycin $(100 \mu \mathrm{g} / \mathrm{ml})]$ at $37^{\circ} \mathrm{C}$ in a humidified atmosphere with $5 \% \mathrm{CO}_{2}$ for $72 \mathrm{~h}$; they were then examined under a microscope.

Statistical analysis. Image programmer software was used to analyze the images. Data are presented as the means \pm standard deviation (SD). Statistical analyses were performed using paired t-tests where applicable. Statistical analysis was performed using SPSS and GraphPad Prism 5 Demo software. A p-value $<0.05$, based on a two-tailed test, was considered to indicate a statistically significant difference.

\section{Results}

Characterization of MSCs. After discarding the non-adherent cells by the first medium change and washing with PBS 3 times at $24 \mathrm{~h}$ of primary culture, approximately $80 \%$ of the MSCs had adhered to the culture dishes; the medium was then changed to remove the suspension of hematopoietic stem cells. After 3 days in primary culture, the MSCs adhered to the plastic surface, presenting a small population of single cells. The cells were spindle-shaped with a single nucleus (Fig. 1A). Seven to 10 days after initial plating, the cells resembled long spindle-shaped fibroblast cells and began to form colonies (Fig. 1B and C). After replating, almost $100 \%$ of the cells had adhered to the culture dishes, and were polygonal or spindle-shaped, with long processes.

The rat MSC surface antigen profiles obtained by flow cytometry (Fig. 2), were positive for CD90, CD29 and negative for CD45. The percentage of CD90 and CD29 was 99.96 and $99.75 \%$, respectively; however, the percentage of CD45 was $1.12 \%$.

Detection of protein expression of nesprin-1 in MSCs by immunofluorescence and western blot analysis. Immunofluorescent staining for nesprin-1 protein verified the presence of rat MSCs (Fig. 3A), with granular green fluorescence distributed around the nuclear membrane of the MSCs. The protein expression of nesprin-1 was detected by western blot analysis (Fig. 3B).

Transfection of MSCs with LV-siNesprin-1, LV-GFP and the detection of protein expression of nesprin-1 by western blot analysis. The MSCs were successfully transfected with LV-siNesprin-1 or LV-GFP, as shown by green fluorescence (Fig. 4A-C). After the MSCs were transfected with LV-siNesprin-1, the protein expression of nesprin-1 in the LV-siNesprin-1 group was lower than that in the LV-GFP and normal group (Fig. 4D). The protein expression of nesprin-1 in the LV-GFP group was the same as that in the normal group, but was significanlty different from that in the LV-siNesprin-1 group ( $\mathrm{P}=0.03$ and $\mathrm{P}=0.028$, respectively; $\mathrm{P}<0.05$ ) (Fig. 4E). The expression of $\beta$-actin did not differ between the 3 groups $(\mathrm{P}=0.10$ and $\mathrm{P}=0.12$, respectively; $\mathrm{P}>0.05)$.

Detection of cell proliferation by MTT assay. The 3 groups of cells (LV-siNesprin-1, LV-GFP and normal group) were 

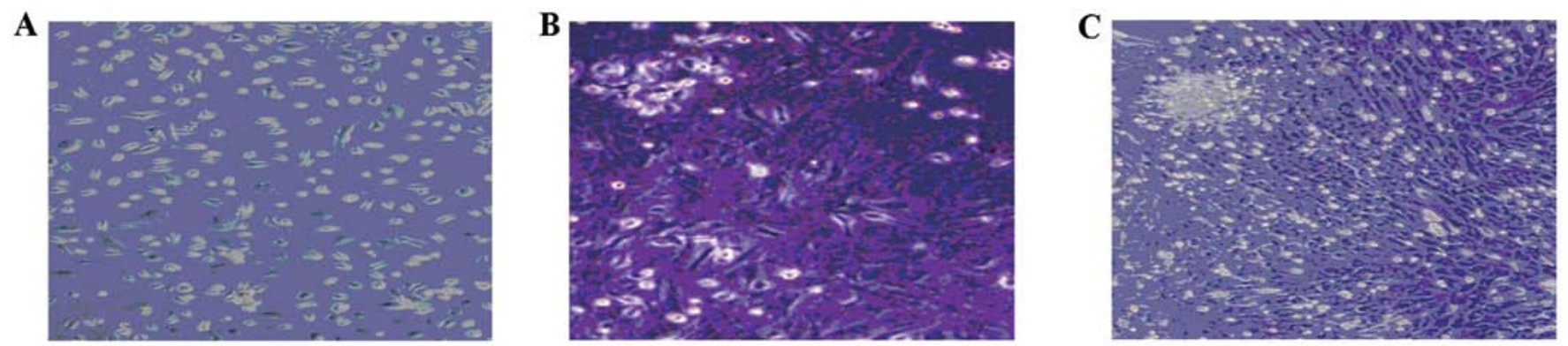

Figure 1. Characterization of mesenchymal stem cells (MSCs) and differentiated cardiomyocyte-like cells in vitro. (A) Morphological observation of rat MSCs after cell culture for 3 days (x100). The cells were spindle-shaped with a single nucleus. (B) Morphological observation of rat MSCs after cell culture for 7 days (x100). (C) Morphological observation of rat MSCs after cell culture for 10 days (x100). The cells had long spindle-shaped fibroblasts and began to form colonies
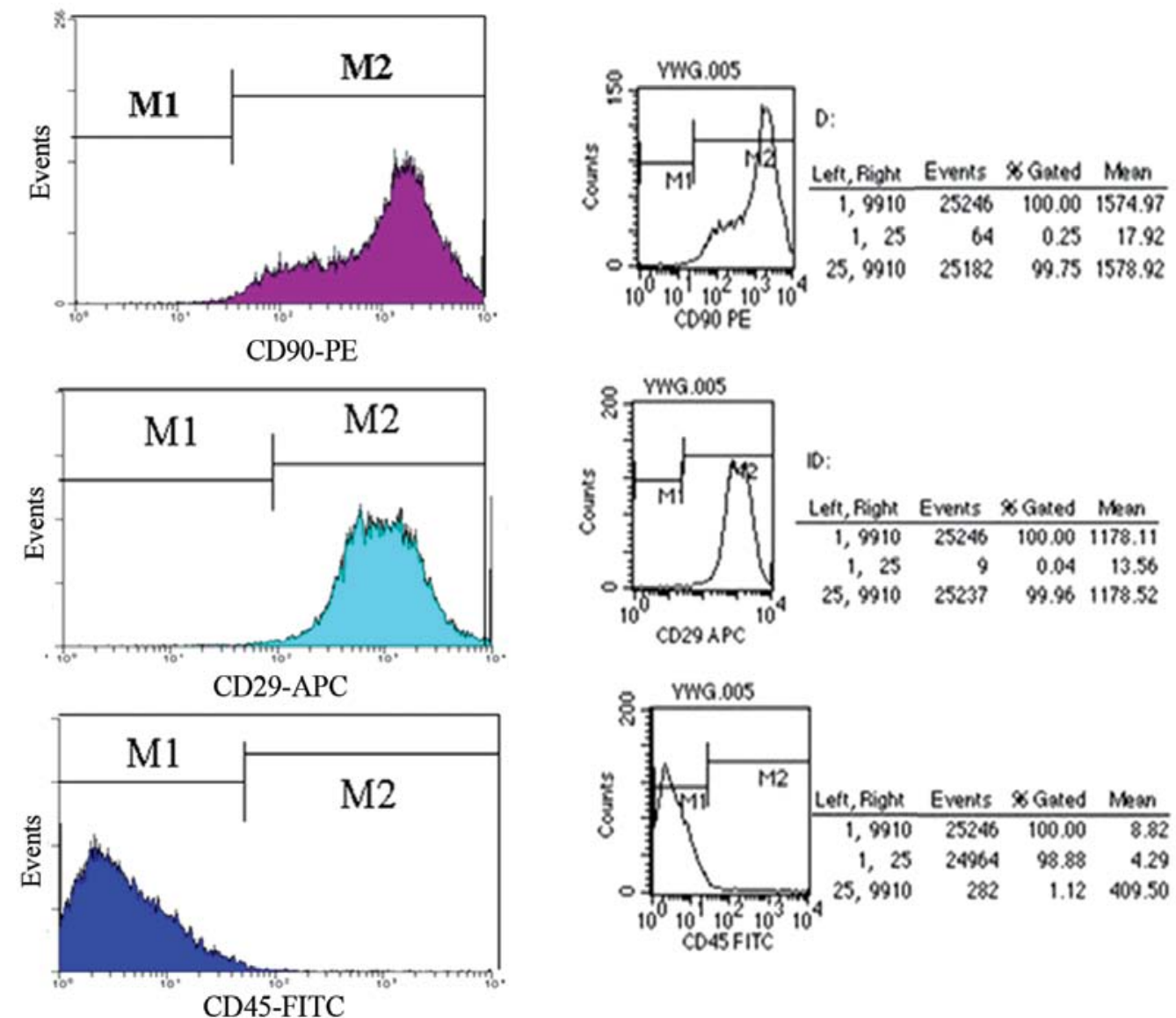

Figure 2. Surface-associated antigens (CD29, CD90, CD45) of MSCs were detected by flow cytometry. MSCs were positive for CD90, CD29 and negative for CD45. The percentage of CD90 and CD29 was approximately 99\%; the percentage of CD45 was approximately $1 \%$.
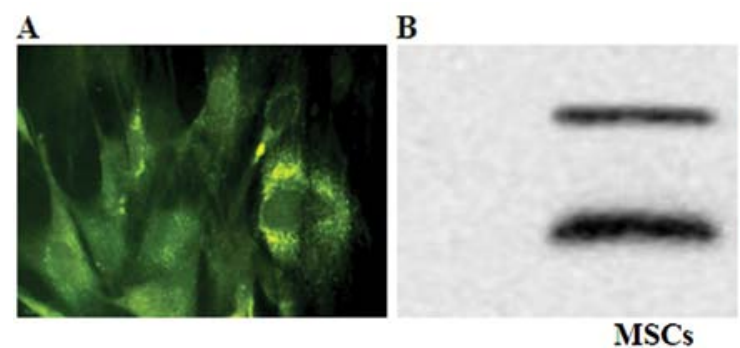

nesprin-1

$\beta$-actin

Figure 3. Immunofluorescence and western blot analysis for the identification of the protein expression of nesprin-1. (A) MSCs were found to be positive for nesprin-1 by immunofluorescence (x400). (B) Western blot analysis revealed the protein expression of nesprin-1 in the MSCs. cultured for 24, 48, 72 and $96 \mathrm{~h}$ under the same conditions. The proliferation of the cells was then detected by MTT assay at 24, 48, 72 and $96 \mathrm{~h}$. As shown in Fig. 5, the knockdown of nesprin-1 expression led to a decrease in the proliferation of MSCs; however, the proliferation rate of the cells in the LV-GFP group was not altered and was similar to the normal group.

Cell cycle of MSCs in the 3 groups (LV-siNesprin-1, LV-GFP and normal group). The 3 groups of cells (LV-siNesprin-1, LV-GFP and normal group) were cultured for $72 \mathrm{~h}$ under the same conditions. The cell cycle was detected by flow cytom- 


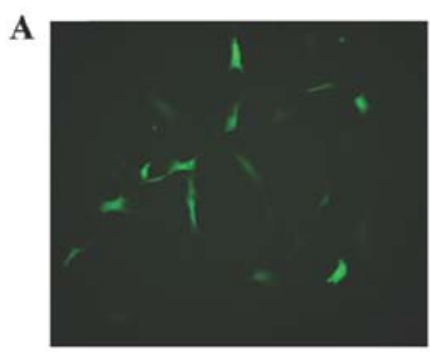

LV-siNesprin-1

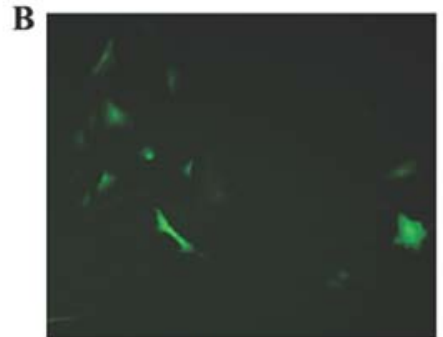

LV-GFP

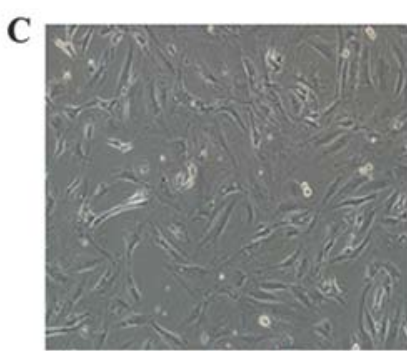

Normal

D

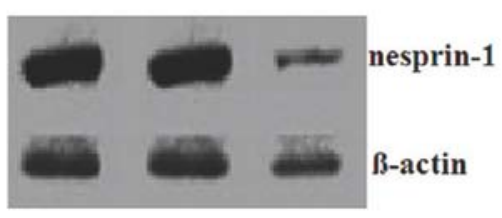

Normal LV-GFP LV-siNesprin-1

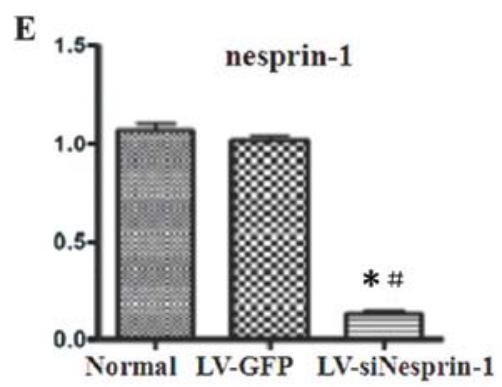

Figure 4. Mesenchymal stem cells (MSCs) were transfected with LV-siNesprin-1 or LV-GFP; the protein expression of nesprin-1 was detected by western blot analysis. (A-C) The MSCS were successfully transfected with LV-siNesprin-1 and LV-GFP (green fluorescence); 200 . (D and E) The protein expression of nesprin-1 in the MSCs in the LV-siNesprin-1 group was lower than that in the MSCS in the LV-GFP and normal group. The protein expression of nesprin-1 in the LV-GFP group was the same as that in the normal group. The difference between the LV-siNesprin-1 group and the LV-GFP/normal group was significant $\left({ }^{*} \mathrm{P}=0.03,{ }^{\prime \prime} \mathrm{P}=0.028\right.$, respectively; $\left.\mathrm{P}<0.05\right)$.
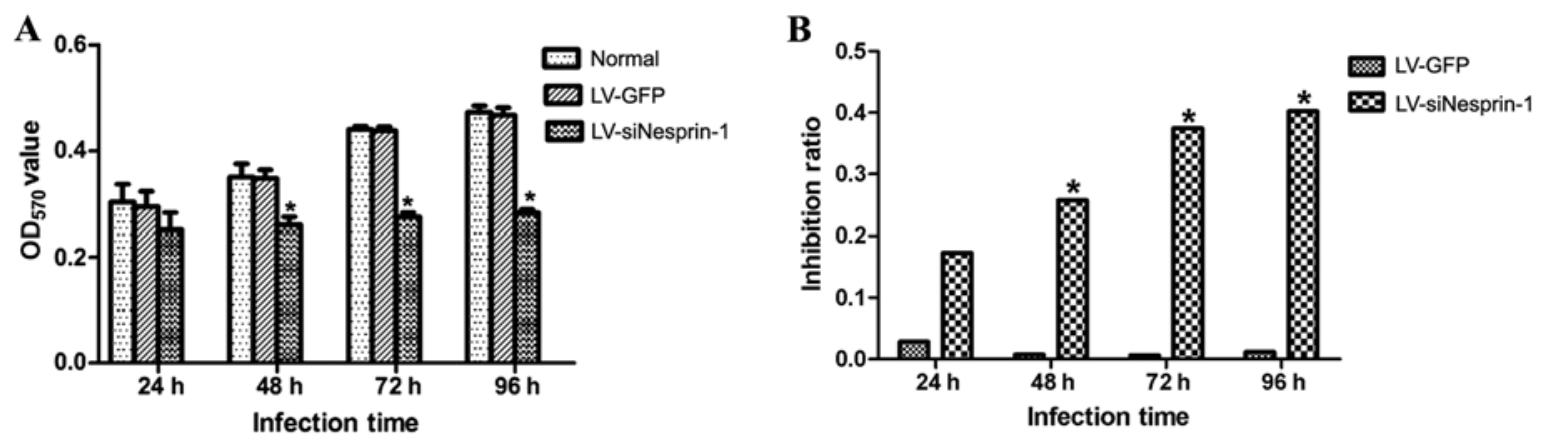

Figure 5. Cell proliferation was detected by MTT assay. (A) Cell proliferation was detected by MTT assay at 24, 48, 72 and $96 \mathrm{~h}$ when the 3 groups of cells (LV-siNesprin-1, LV-GFP and normal group) were cultured for 24, 48, 72 and $96 \mathrm{~h}$ under the same conditions. The proliferation of the cells in the LV-siNesprin-1 group was reduced compared with the other 2 groups; this difference was statistically significant $($ ( $\mathrm{P}=0.025 ; \mathrm{P}<0.05)$. However, the proliferation of the cells in the LV-GFP group was the same as that in the normal group. (B) Transfection with LV-siNesprin-1 inhibited cell proliferation; however, transfection with LV-GFP did not affect cell proliferation.

etry. In the LV-siNesprin-1 group, the cells were mainly in the G0/G1 phase of the cell cycle (Fig. 6).

Apoptosis of MSCs in the 3 groups (LV-siNesprin-1, LV-GFP and normal group). The 3 groups of cells (LV-siNesprin-1, LV-GFP and normal group) were cultured for $72 \mathrm{~h}$ under the same conditions. The apoptosis of the cells was detected by flow cytometry. In the LV-siNesprin-1 group, the apoptotic rate of the cells was higher than that of the cells in the LV-GFP and normal group (Fig. 7).

Changes in the morphology of the nucleus following transfection of MSCs with LV-siNesprin-1. As shown in Fig. 8, the morphology of the nucleus in the LV-siNesprin-1 group was altered; morphological changes such as fusion and fragmentation were observed.

\section{Discussion}

Nesprins have been reported to bind to the nuclear membrane. Through interaction with both emerin and lamin $\mathrm{A} / \mathrm{C}$, nesprin is localized to the nuclear membrane $(2,4,5,21)$. The structure of the various nesprin isoforms suggests that they also form a protein scaffold that links the nuclear membrane with the nucleus, cytoplasmic organelles and the cell membrane via the actin cytoskeleton. Nesprin (C. elegans ANC-1) mutations may disrupt the positioning of the mitochondria in mononuclear cells (22). These studies suggest a structural role for nesprins in nuclear migration and the positioning of major organelles (23). Nesprin (Drosophila MSP-300) has been shown to be localized to regions of the cell margin involved in exoskeletal attachment and to the Z-lines in sarcomeres. These studies suggest its role in the structural organization of the sarcomere 

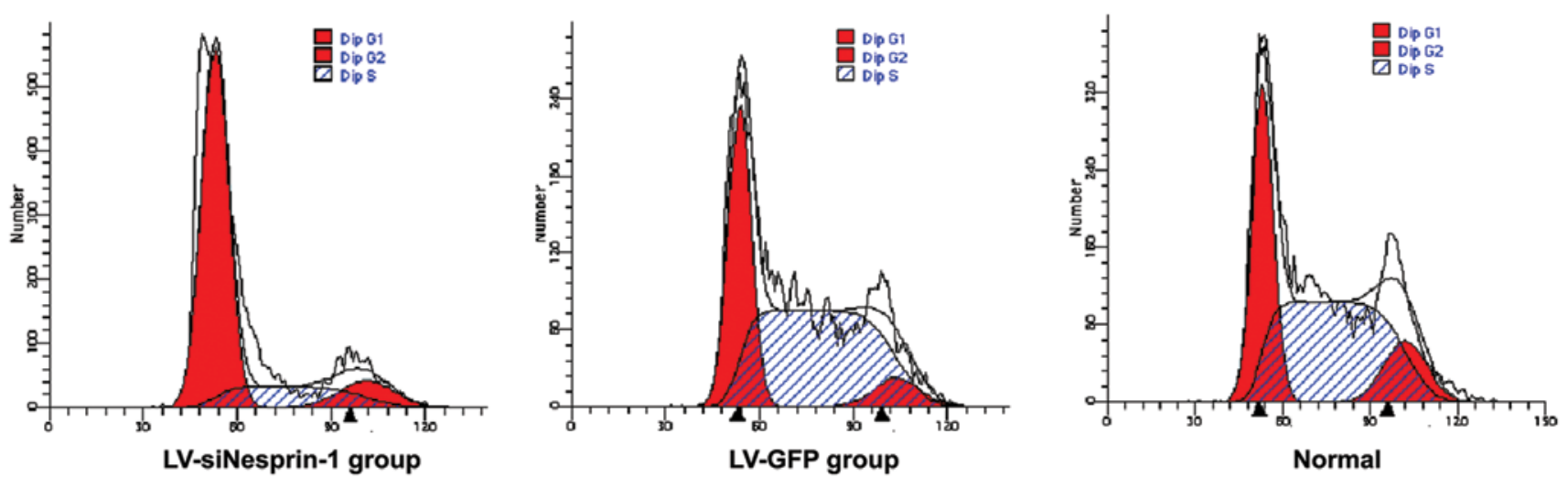

Figure 6. Cell cycle of mesenchymal stem cell (MSC) in the 3 groups (LV-siNesprin-1, LV-GFP and normal group) analyzed by flow cytometry. The 3 groups of cells (LV-siNesprin-1, LV-GFP and normal group) were cultured for $72 \mathrm{~h}$ under the same conditions. Cell cycle was then analyzed by flow cytometry. The differentiation of cells in the LV-siNesprin-1 group was slower than that of cells in the LV-GFP and normal group; the cells were mainly in the G0/G1 phase of the cell cycle, (approximately $68.45 \pm 3.75 \%$ ). In the LV-GFP and normal group, the percentage of cells in this phase was approximately $40.57 \pm 2.45$ and $42.17 \pm 2.05 \%$, respectively. The difference between the LV-siNesprin-1 group and the normal group was statistically significant $(\mathrm{P}=0.035 ; \mathrm{P}<0.05)$.
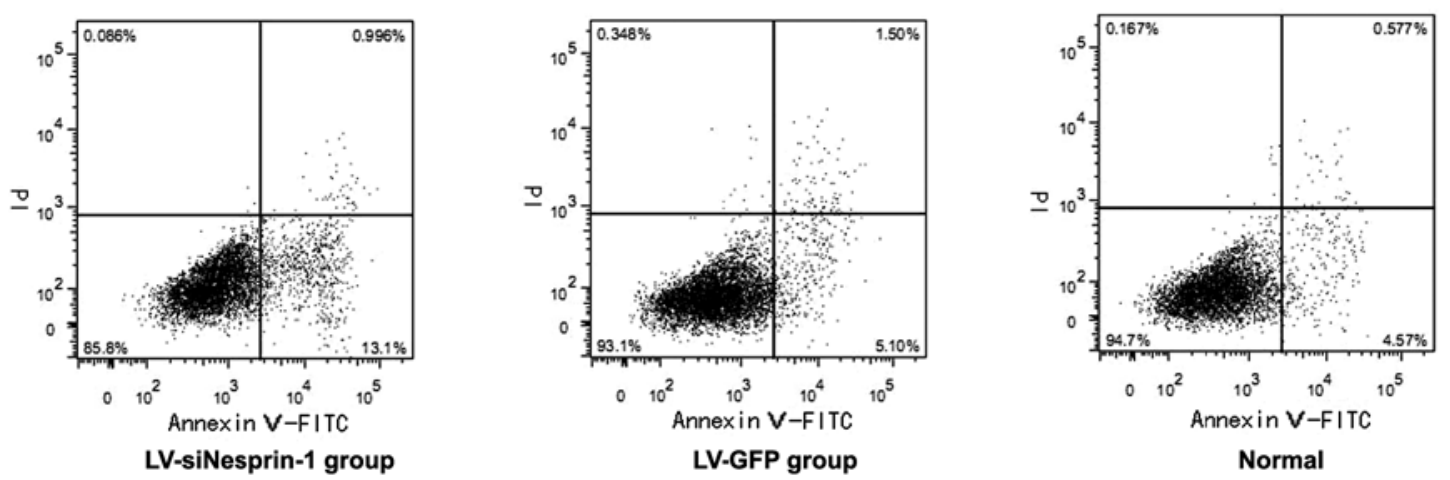

Figure 7. Cell apoptosis of mesenchymal stem cells (MSCs) in the 3 groups (LV-siNesprin-1, LV-GFP and normal group) detected by flow cytometry. The 3 groups of cells (LV-siNesprin-1, LV-GFP and normal group) were cultured for $72 \mathrm{~h}$ under the same conditions. The cell cycle was analyzed by flow cytometry. The apoptotic rate of the cells in the LV-siNesprin-1 group was higher than that of cells in the LV-GFP and normal group; the percentage of apoptotic cells in the LV-siNesprin-1 group was approximately $15.12 \pm 0.97 \%$ at $72 \mathrm{~h}$. The percentage of apoptotic cells in the LV-GFP and normal group was approximately $6.86 \pm 1.26$ and $5.04 \pm 0.80 \%$, respectively. The difference between the LV-siNesprin-1 group and the LV-GFP/normal group was statistically significant $(\mathrm{P}=0.032, \mathrm{P}=0.025$, respectively; $\mathrm{P}<0.05)$.

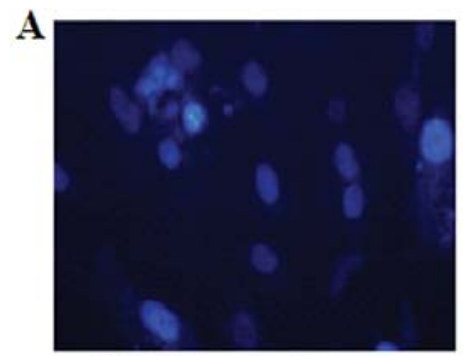

LV-siNesprin-1 group

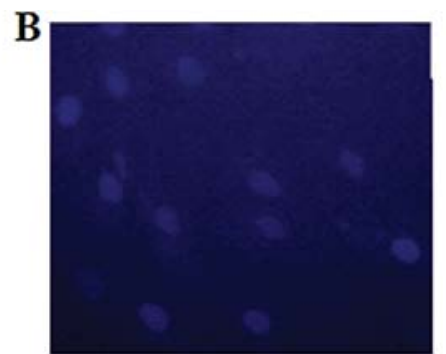

LV-GFPgroup

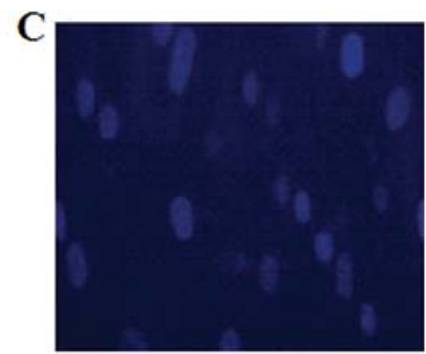

Normal group

Figure 8. The morphology of the nucleus in the mesenchymal stem cells (MSCs) was observed $72 \mathrm{~h}$ after transfection and staining with DAPI. (A) The morphology of the nucleus in the LV-siNesprin-1 group was altered; morphological changes such as fusion and fragmentation were observed (x200). (B) The morphology of the nucleus in the LV-GFP group was not altered (x200). (C) The morphology of the nucleus in the normal group (x200).

and in signalling from the extracellular environment to the nucleus. Gough et al (24) concluded that the nesprin-1 gene is expressed in a variety of forms that are multifunctional and are capable of functioning at both the Golgi and the nuclear envelope, with the linking of the 2 organelles taking place during muscle cell differentiation.
Nesprins are widely expressed in a variety of tissues; the high expression of both nesprin-1 and -2 has been observed in skeletal, cardiac and vascular smooth muscls. Zhang et al (2) suggested that nesprins have a specific function in muscle cell differentiation. However, the high expression of nesprin-1 has also been found in peripheral blood leukocytes and spleen, 
and nesprin-2 in the pancreas and testes. Nesprins are highly expressed in muscles with both nesprin-1 and -2 muscle-specific isoforms $(2,21,25)$. In vitro, during the differentiation of $\mathrm{C} 2 \mathrm{C} 12$ myoblasts into myotubes, nesprin changes its localization from the nuclear membrane to the cytoplasm/sarcomere, indicating its specific roles during muscle differentiation $(2,4)$. In the sarcomere of skeletal muscle cells, different nesprin-1 and -2 epitopes are associated with the Z-line, the A/I junction, the sarcoplasmic reticulum and the mitochondrial membrane, indicating that nesprins may play the role of maintaining sarcomeric structure $(4,25)$. In addition, sarcomeric proteins have been identified as potential interacting partners for nesprins, including the ryanodine receptor and $\operatorname{mAKAP}(25,26)$. mAKAP is targeted to the nuclear membrane by nesprin-1 and they interact through their closely related spectrin repeats (26). Potentially, nesprins may be involved in maintaining and/ or targeting protein complexes common to both the nuclear membrane and the sarcoplasmic reticulum (25). Cardiomyocyte nuclei have been found to be elongated in $\Delta / \Delta \mathrm{KASH}$ mouse hearts. These findings reflect what has been reported on lamin $\mathrm{A} / \mathrm{C}$ gene mutations and reinforce the importance of an intact nuclear membrane complex for normal heart function (6). It has been shown that $\Delta / \Delta \mathrm{KASH}$ mice (lacking the carboxy-terminus of nesprin-1) develop cardiomyopathy with associated cardiac conduction system disease. Older mutant animals have been shown to have elongated $\mathrm{P}$ wave duration, elevated atrial and ventricular effective refractory periods, indicating conduction system defects in the myocardium. It has been found that cardiomyocyte nuclei are elongated with reduced heterochromatin in $\Delta / \Delta \mathrm{KASH}$ mouse hearts (6).

The abovementioned data indicate that nesprin seems to play a key role in adult cell mitosis, RNA transport and the stability of the nuclear membrane. Yet, little is known about the role of the nesprin-1 protein, particularly nesprin-1 in stem cells (MSCs). In the present study, we aimed to elucidate the function of nesprin-1 protein in BMSCs by designing a nesprin-1 siRNA lentiviral vector. Following the transfection of the MSCs with LV-siNesprin-1, we found that the protein expression of nesprin-1 in the LV-siNesprin-1 group was lower than that in the LV-GFP and normal group; the proliferation of the MSCs in the LV-siNesprin-1 group was also reduced. However, the apoptotic rate was increased in the LV-siNesprin-1 group compared with the LV-GFP and normal group. As shown by morphological analysis using a microscope, in the LV-siNesprin-1 group, morphological changes were observed in the nucleus, such as fusion and fragmentation. Thus, nesprin-1 mediates cell differentiation and regulates the proliferation and apoptosis of MSCs.

MSCs were first described in 1968 by Friedenstein et al (27). These cells can be expanded and induced, either in vitro or in vivo, and terminally differentiate into osteoblasts, chondrocytes, adipocytes, tenocytes, myotubes, neuronal cells and hematopoietic cells with strong self-renewal ability and genetic stability in vitro. Several research groups have reported that MSCs are able to proliferate and potentially differentiate in vitro (28-30). However, the ratio of MSCs in bone marrow cells is very low, approximately $0.001-0.01 \%$; hence, the separation and amplification of MSCs is of vital significance. Wakitani et al (31) described a method of isolating MSCs from rat bone marrow with Ficoll density gradient separation and adherent culture. Currently, the International Society for Cellular Therapy proposed 3 minimal criteria for defining MSCs (32): i) MSCs must be plastic-adherent if maintained in standard culture conditions; ii) MSCs must express CD105, CD73 and CD90, but must not express haematopoietic markers, such as CD45, CD34, CD14 or CD11b; and iii) MSCs must be capable of differentiating into fibroblasts, osteoblasts, adipocytes and chondroblasts under the corresponding lineage, particularly in in vitro conditions. In this study, we detected the MSC surface antigens, CD90 and CD29, by flow cytometry, with the percentage of CD90 and CD29 as high as 98\%, and the percentage of CD45 approximately $1 \%$.

$\mathrm{Xu}$ et al (33) reported that the ability of human MSCs to proliferate remained strong between passages 2 and 6 , then the apoptosis of MSCs began to accelerate. In the process of MSC proliferation and differentiation, the upregulation of the protein expression of nesprin-1 may maintain the stability of the MSC nuclear membrane and reduce the apoptosis of the MSCs; this may provide a theoretical basis and gain more seed cells for the improvement of therapeutic modalities for heart disease.

\section{Acknowledgements}

This study was supported by a grant from the Municipal Committee of Science and Technology of Shanghai, China (no. 064119636).

\section{References}

1. Lammerding J, Schulze PC, Takahashi T, et al: Lamin A/C deficiency causes defective nuclear mechanics and mechanotransduction. J Clin Invest 113: 370-378, 2004.

2. Zhang Q, Skepper JN, Yang F, et al: Nesprins: a novel family of spectrin-repeat-containing proteins that localize to the nuclear membrane in multiple tissues. J Cell Sci 114: 4485-4498, 2001.

3. Apel ED, Lewis RM, Grady RM and Sanes JR: Syne-1, a dystrophin- and Klarsicht-related protein associated with synaptic nuclei at the neuromuscular junction. J Biol Chem 275: 31986-31995, 2000.

4. Zhang Q, Ragnauth CD, Skepper JN, et al: Nesprin-2 is a multi-isomeric protein that binds lamin and emerin at the nuclear envelope and forms a subcellular network in skeletal muscle. J Cell Sci 118: 673-687, 2005.

5. Mislow JM, Kim MS, Davis DB and McNally EM: Myne-1, a spectrin repeat transmembrane protein of the myocyte inner nuclear membrane, interacts with lamin A/C. J Cell Sci 115: 61-70, 2002.

6. Puckelwartz MJ, Kessler EJ, Kim G, et al: Nesprin-1 mutations in human and murine cardiomyopathy. J Mol Cell Cardiol 48: 600-608, 2010.

7. Pittenger MF, Mackay AM, Beck SC, et al: Multilineage potential of adult human mesenchymal stem cells. Science 284: 143-147, 1999.

8. Minguell JJ, Erices A and Conget P: Mesenchymal stem cells. Exp Biol Med (Maywood) 226: 507-520, 2001.

9. Devine SM: Mesenchymal stem cells: will they have a role in the clinic? J Cell Biochem Suppl 38: 73-79, 2002.

10. Nagaya N, Fujii T, Iwase T, et al: Intravenous administration of mesenchymal stem cells improves cardiac function in rats with acute myocardial infarction through angiogenesis and myogenesis. Am J Physiol Heart Circ Physiol 287: H2670-H2676, 2004.

11. Miyahara Y, Nagaya N, Kataoka M, et al: Monolayered mesenchymal stem cells repair scarred myocardium after myocardial infarction. Nat Med 12: 459-465, 2006.

12. Nagaya N, Kangawa K, Itoh T, et al: Transplantation of mesenchymal stem cells improves cardiac function in a rat model of dilated cardiomyopathy. Circulation 112: 1128-1135, 2005.

13. Naldini L, Blömer U, Gallay P, et al: In vivo gene delivery and stable transduction of nondividing cells by a lentiviral vector. Science 272: 263-267, 1996. 
14. Dull T, Zufferey R, Kelly M, et al: A third-generation lentivirus vector with a conditional packaging system. J Virol 72: 8463-8471, 1998.

15. Guenechea G, Gan OI, Inamitsu T, et al: Transduction of human $\mathrm{CD} 34^{+} \mathrm{CD} 38^{-}$bone marrow and cord blood-derived SCID-repopulating cells with third-generation lentiviral vectors. Mol Ther 1: 566-573, 2000.

16. Naldini L, Blömer U, Gage FH, Trono D and Verma IM: Efficient transfer, integration, and sustained long-term expression of the transgene in adult rat brains injected with a lentiviral vector. Proc Natl Acad Sci USA 93: 11382-11388, 1996.

17. Kafri T, Blömer U, Peterson DA, Gage FH and Verma IM: Sustained expression of genes delivered directly into liver and muscle by lentiviral vectors. Nat Genet 17: 314-317, 1997.

18. Vermes I, Haanen C, Steffens-Nakken $\mathrm{H}$ and Reutelingsperger C: A novel assay for apoptosis. Flow cytometric detection of phosphatidylserine expression on early apoptotic cells using fluorescein labelled Annexin V. J Immunol Methods 184: 39-51, 1995.

19. Koopman G, Reutelingsperger CP, Kuijten GA, Keehnen RM, Pals ST and van Oers MH: Annexin V for flow cytometric detection of phosphatidylserine expression on B cells undergoing apoptosis. Blood 84: 1415-1420, 1994

20. Park C, Moon DO, Choi IW, et al: Curcumin induces apoptosis and inhibits prostaglandin $\mathrm{E}_{2}$ production in synovial fibroblasts of patients with rheumatoid arthritis. Int J Mol Med 20: 365-372, 2007.

21. Mislow JM, Holaska JM, Kim MS, et al: Nesprin-1alpha self-associates and binds directly to emerin and lamin A in vitro. FEBS Lett 525: 135-140, 2002.

22. Starr DA and Han M: Role of ANC-1 in tethering nuclei to the actin cytoskeleton. Science 298: 406-409, 2002.

23. Starr DA and Han M: ANChors away: an actin based mechanism of nuclear positioning. J Cell Sci 116: 211-216, 2003.
24. Gough LL, Fan J, Chu S, Winnick S and Beck KA: Golgi localization of Syne-1. Mol Biol Cell 14: 2410-2424, 2003.

25. Zhang Q, Ragnauth C, Greener MJ, Shanahan CM and Roberts RG: The nesprins are giant actin-binding proteins, orthologous to Drosophila melanogaster muscle protein MSP-300. Genomics 80: 473-481, 2002.

26. Pare GC, Easlick JL, Mislow JM, McNally EM and Kapiloff MS: Nesprin-1alpha contributes to the targeting of mAKAP to the cardiac myocyte nuclear envelope. Exp Cell Res 303: 388-399, 2005.

27. Friedenstein AJ, Petrakova KV, Kurolesova AI and Frolova GP: Heterotopic of bone marrow. Analysis of precursor cells for osteogenic and hematopoietic tissues. Transplantation 6: 230-247, 1968.

28. Dennis JE and Charbord P: Origin and differentiation of human and murine stroma. Stem Cells 20: 205-214, 2002.

29. Campagnoli C, Roberts IA, Kumar S, Bennett PR, Bellantuono I and Fisk NM: Identification of mesenchymal stem/progenitor cells in human first-trimester fetal blood, liver, and bone marrow. Blood 98: 2396-2402, 2001.

30. Martin DR, Cox NR, Hathcock TL, Niemeyer GP and Baker HJ: Isolation and characterization of multipotential mesenchymal stem cells from feline bone marrow. Exp Hematol 30: 879-886, 2002.

31. Wakitani S, Saito T and Caplan AI: Myogenic cells derived from rat bone marrow mesenchymal stem cells exposed to 5-azacytidine. Muscle Nerve 18: 1417-1426, 1995.

32. Dominici M, Le Blanc K, Mueller I, et al: Minimal criteria for defining multipotent mesenchymal stromal cells. The International Society for Cellular Therapy position statement. Cytotherapy 8: 315-317, 2006.

33. Xu W, Zhang X, Qian H, et al: Mesenchymal stem cells from adult human bone marrow differentiate into a cardiomyocyte phenotype in vitro. Exp Biol Med (Maywood) 229: 623-631, 2004. 